\title{
PERFORMANCE COMPARISON OF INSTITUTIONAL AND RETAIL MUTUAL FUNDS USING MULTIFACTOR MODELS
}

\author{
Mehmet Sencicek \\ The BRC Academy Journal of Business 10, no. 1 (2020): 1-31. \\ https://dx.doi.org/10.15239/j.brcacadjb.2020.10.01.ja01
}

Web Appendix

https://dx.doi.org/10.15239/j.brcacadjb.2020.10.01.wa01 
Performance Comparison of Institutional and Retail Mutual Funds using

\section{Models}

\section{APPENDIX A. Tables for measures of fund performance and differences in measures.}

The following tables show performance comparison results with each model. The regression equations referenced in the study for the models used are as follows:

$$
\begin{array}{ll}
\text { CAPM }: & \mathrm{R}_{\mathrm{it}}-\mathrm{R}_{\mathrm{ft}}=\alpha_{\mathrm{i}}+\beta_{\mathrm{i}}\left(\mathrm{R}_{\mathrm{mt}}-\mathrm{R}_{\mathrm{ft}}\right)+\varepsilon_{\mathrm{t}}, \\
\text { FF3F } & : \mathrm{R}_{\mathrm{it}} \mathrm{R}_{\mathrm{ft}}=\alpha_{\mathrm{i}}+\beta_{0 \mathrm{i}}\left(\mathrm{R}_{\mathrm{mt}}-\mathrm{R}_{\mathrm{ft}}+\beta_{1 \mathrm{i}} \mathrm{SMB}+\beta_{2 \mathrm{i}} \mathrm{HML}+\varepsilon_{\mathrm{it}}\right. \\
\mathrm{C} 4 \mathrm{~F} \quad: \mathrm{R}_{\mathrm{it}} \mathrm{R}_{\mathrm{ft}}=\alpha_{\mathrm{i}}+\beta_{0 \mathrm{i}}\left(\mathrm{R}_{\mathrm{mt}}-\mathrm{R}_{\mathrm{ft}}+\beta_{1 \mathrm{i}} \mathrm{SMB}+\beta_{2 \mathrm{i}} \mathrm{HML}+\beta_{3 \mathrm{i}} \mathrm{MOM}+\varepsilon_{\mathrm{it}}\right.
\end{array}
$$

where

$\beta_{\mathrm{i}}$ and $\beta_{0 \mathrm{i}}$ are fund betas, in the CAPM and the multifactor models, respectively,

$\mathrm{R}_{\mathrm{it}}$ and $\mathrm{R}_{\mathrm{ft}}$ are a fund's monthly return ${ }^{1}$, the monthly risk-free rate ${ }^{2}$ at time $t$,

$\mathrm{R}_{\mathrm{it}} \mathrm{R}_{\mathrm{ft}}$ is fund's risk premium at time $t$,

$\mathrm{R}_{(\mathrm{mt})}$ is the monthly return on the fund's benchmark at time $t$,

$\mathrm{R}_{(\mathrm{mt})}-\mathrm{R}_{(\mathrm{ft})}$ is the market risk premium at time $t$,

$\mathrm{SMB}_{t}{ }_{t}$ is the difference in return between a small cap portfolio and a large cap portfolio at time $t$,

$\mathrm{HML}_{t}$ is the difference in return at time $t$ between a portfolio containing 'value' stocks (with a high book-to-market ratio) and one consisting of 'growth' stocks with a (low book-to-market ratio).

CAPM, FF3F and C4F are Capital Asset Pricing Model, Fama-French Three-Factor Model and Carhart Four-Factor model, respectively.

In the following tables, "difference" is simply the difference between the slope coefficients of institutional and retail funds for a given category obtained by least squares regression (equations (4), (5) and (6) above). The significance of the difference in regression coefficients in each category was tested by computing the $p$ value for the test statistic for the difference $\left(=\mathrm{d} / \mathrm{se}_{\mathrm{d}}\right)$ where $\mathrm{d}$ is the difference in slopes $\left(=b_{1}-b_{2}\right)$, and $s_{d}$ is the standard error of the difference; without assuming homogeneity of the error variances and with large samples (each $\mathrm{n}>25), \mathrm{se}_{\mathrm{d}}=\sqrt{ }\left(\left(\mathrm{s}_{\mathrm{b} 1}\right)^{2}+\left(\mathrm{s}_{\mathrm{b} 2}\right)^{2}\right.$, where $\mathrm{s}_{\mathrm{b} 1}, \mathrm{~s}_{\mathrm{b} 2}$ are the standard errors of the slope coefficients $b_{1}$ and $b_{2}$, respectively. Statistically significant coefficients or differences are indicated by $\left({ }^{* * *}\right),\left(^{* *}\right)$ or $\left(^{*}\right)$ depending on the level of significance specified below each of the following tables. 
Table A-1. Overall performance comparisons results with each model.

(a) Results with CAPM

$\begin{array}{lccc}\text { Fund class } & \text { Alpha } & \text { Market } & \mathbf{R}^{2}{ }_{\text {ad }} \\ \text { Institutional } & 0.142 & 1.070^{* * *} & 0.854 \\ \text { Retail } & 0.074 & 1.014^{* * *} & 0.873 \\ \text { Difference } & 0.068^{*} & 0.056 & -0.020\end{array}$

(b) Results with Fama-French Three Factor Model

$\begin{array}{lccccc}\text { Fund class } & \text { Alpha } & \text { Market } & \text { SMB } & \text { HML } & \mathbf{R}_{\text {adj }}^{2} \\ \text { Institutional } & 0.132 & 1.029^{* * *} & 0.232^{* * *} & 0.002 & 0.859 \\ \text { Retail } & 0.096 & 0.964^{* * *} & 0.316^{* * *} & -0.028 & 0.890 \\ \text { Difference } & 0.036 & 0.065 & -0.084^{*} & 0.031 & -0.031\end{array}$

(c) Results with Carhart Four Factor Model

\begin{tabular}{|c|c|c|c|c|c|c|c|}
\hline Fund class & Alpha & Market & & SMB & HML & Mom & $\mathbf{R}_{\text {adj }}^{2}$ \\
\hline Institutional & 0.133 & 1.003 & $* * *$ & $0.236^{* * *}$ & $-0.057^{* *}$ & $-0.090^{*}$ & 0.864 \\
\hline Retail & 0.096 & 0.942 & ${ }^{* * *}$ & $0.319^{*}$ & -0.079 & $-0.077^{*}$ & 0.894 \\
\hline Difference & 0.037 & 0.061 & & $-0.083^{*}$ & 0.022 & -0.013 & -0.030 \\
\hline
\end{tabular}

Table A-2. Results by size categories with each model.

(a) Results by size categories with CAPM

Fund

category/class Alpha Market $\quad \mathbf{R}^{2}{ }_{\text {adj }}$ 


\section{Large Cap}

Institutional

$\begin{array}{lll}0.238^{*} & 0.935^{* * *} & 0.894 \\ 0.030 & 0.971^{* * *} & 0.896 \\ 0.208^{* *} & -0.036^{*} & -0.003\end{array}$

Difference

\section{Midcap}

$\begin{array}{lccc}\text { Institutional } & 0.134 & 1.028^{* * *} & 0.807 \\ \text { Retail } & 0.165 & 1.035^{* * *} & 0.714 \\ \text { Difference } & -0.030 & -0.008 & 0.093\end{array}$

\section{Small Cap}

Institutional

0.171

$0.849^{* * *}$

0.787

Retail

0.145

$0.889^{* * *}$

0.895

Difference

0.026

$-0.041$

$-0.108$

(b) Results by size categories with the Fama-French Three Factor Model

\section{Fund}

category/class

Alpha Market

SMB

HML

$\mathbf{R}_{\text {adj }}^{2}$

\section{Large Cap}

Institutional

$0.223^{*} \quad 0.949^{* * *}$

$0.120 *$

$-0.018^{* *}$

0.895

Retail

0.015

$0.979^{* * *}$

$0.026^{*}$

$-0.059$

0.882

Difference

$0.208^{* *}$

$-0.029^{*}$

0.094

0.041

0.896 


\section{Midcap}

Institutiona

$0.090 \quad 1.009^{* * *} \quad 0.120^{* *} \quad-0.018^{*} \quad 0.819$

Retail

$0.110^{*}$

$1.009^{* * *}$

$0.335^{*}-0.155$

0.882

Difference

$-0.020$

0.000

$-0.215^{*} \quad 0.137$

0.725

\section{Small Cap}

Institutional
0.166 *
$0.871^{* * *}$
$0.120^{* * *}-0.018$
0.784

Retail

0.114

$0.924^{* * *}$

$-0.076^{* * *}-0.083$

0.882

Difference

0.053

$-0.053$

0.196

0.065

0.895

(c) Results by size categories with Carhart Four Factor Model

$\begin{array}{lllllll}\text { Fund/category } & \text { Alpha } & \text { Market } & \text { SMB } & \text { HML } & \text { MOM } & \mathbf{R}^{2}{ }_{\text {adj }}\end{array}$

\section{Large Cap}

Institutional

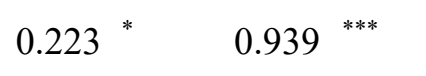

$-0.052^{*}$

$-0.131^{* * *}$

$-0.074^{* *}$

0.900

Retail

0.016

$0.958^{* * *} \quad 0.028$

$-0.105$

$-0.069$

0.899

Difference

$0.207^{* *}$

$-0.019^{* *}$

$-0.080^{* *}$

$-0.027^{* *}$

$-0.005^{* *}$

0.001

\section{Midcap}

Institutional

0.091

$0.983^{* * *}$

$0.264^{* * *}$

$-0.198^{*}$

$-0.090^{*}$

0.824

Retail

0.108

$0.996^{* * *}$

$0.336^{*}$

$-0.197$

$-0.060^{*}$

0.724

Difference

$-0.017$

$-0.013$

$-0.071$

$-0.001$

$-0.030$

0.099

\section{Small Cap}

Institutional

$$
0.167^{*}
$$

$0.836^{* * *}$

$-0.046^{* * *}-0.095$

$-0.134^{* *}$

0.794

Retail

$0.114^{*}$

$0.899^{* * *}$

$-0.051^{* * *}-0.144$

$-0.096^{* *}$

0.901 
Table A-3. Results by book-to-market ratio (BM) categories.

(a) Results by BM categories with CAPM

Fund

category/class Alpha Market $\quad \mathbf{R}^{2}{ }_{\text {adj }}$

Value

Institutional $\quad 0.179^{*} \quad 0.969^{* * *} \quad 0.893$

Retail $\quad 0.243^{*} \quad 1.009^{* * *} \quad 0.874$

Difference $\quad-0.064 \quad-0.039 \quad 0.019$

Blend

Institutional $\quad 0.173^{*} \quad 0.988^{* * *} \quad 0.922$

Retail $\quad 0.095 \quad 1.003^{* * *} \quad 0.867$

Difference $\quad 0.078 \quad-0.015 \quad 0.054$

\section{Growth}

Institutional $\quad 0.029 \quad 1.071^{* * *} \quad 0.803$

Retail $0.058 \quad 1.013^{* * *} \quad 0.831$

Difference $\quad-0.029 \quad 0.058 \quad-0.028$

(b) Results by BM categories with the Fama-French Three Factor Model

\section{Fund}

category/class Alpha Market $\quad$ SMB $\quad$ HML $\quad \mathbf{R}_{\text {adj }}^{2}$


Value

Institutional

$0.178^{*} \quad 0.941^{* * *}$

$0.120^{* * *}-0.018^{* * *}$

0.894

Retail

$0.202^{*}$

$0.967^{* * *}$

$0.352^{* * *}-0.062^{* *}$

0.882

Difference

$-0.024$

$-0.025$

$-0.232$

0.043

0.895

\section{Blend}

Institutional

$$
0.163^{*}
$$

$0.970^{* * *}$

$0.120^{* *} \quad-0.018$

0.923

Retail

0.084

$0.949^{* * *}$

$0.293^{* * *} \quad 0.013$

0.882

Difference

0.079

0.022

$-0.173^{* *}-0.032$

0.882

\section{Growth}

$\begin{array}{lccccc}\text { Institutional } & 0.005 & 1.021^{* * *} & 0.120^{* * *} & -0.018^{* * *} & 0.820 \\ \text { Retail } & 0.046 & 0.958^{* * *} & 0.329^{* * *} & -0.013^{* * *} & 0.882 \\ \text { Difference } & -0.041 & 0.063 & -0.208 & -0.005 & 0.848\end{array}$

(c) Results by BM categories with the Carhart Four Factor Model

$\begin{array}{llllll}\text { Fund/category } & \text { Alpha } & \text { Market } & \text { SMB } & \text { HML } & \text { MOM }\end{array}$

\begin{tabular}{lcccccc} 
Value & & & & \\
Institutional & $0.178^{*}$ & $0.906^{* * *}$ & $0.133^{* * *}$ & $-0.067^{*}$ & $-0.136^{* *}$ & 0.907 \\
Retail & $0.200^{*}$ & $0.933^{* * *}$ & $0.356^{* * *}$ & -0.125 & $-0.111^{* * *}$ & 0.903 \\
\hline Difference & -0.022 & $-0.027^{* *}$ & -0.223 & 0.059 & -0.025 & 0.003
\end{tabular}

\section{Blend}

Institutional

$0.164^{*} \quad 0.954^{* * *}$

$0.123^{*}$

$-0.055$

$-0.056^{* *} \quad 0.925$ 


\begin{tabular}{lcccccr} 
Retail & 0.085 & $0.929^{* *}$ & $0.296^{* * *}$ & -0.031 & $-0.067^{* *}$ & 0.885 \\
\hline Difference & 0.079 & 0.025 & -0.174 & -0.024 & 0.011 & 0.041
\end{tabular}

\section{Growth}

\begin{tabular}{|c|c|c|c|c|c|c|c|}
\hline Institutional & 0.008 & 1.003 & *** & $0.326^{* * *}$ & $-0.134^{* * *}$ & -0.057 & 0.821 \\
\hline Retail & 0.048 & 0.941 & *** & $0.332^{* * *}$ & $-0.057^{* * *}$ & -0.060 & 0.849 \\
\hline Difference & -0.041 & 0.062 & & -0.005 & -0.077 & $0.003^{* *}$ & -0.029 \\
\hline
\end{tabular}

\section{APPENDIX B. Fund Categories and benchmarks}

Morningtar's style classification allocates funds into different categories based on fund's investment methodology and the market capitalization of the companies in which it invests. It places the funds in large-cap, medium-cap or small-cap based on the market capitalization of the equities they hold. Funds are characterized as growth oriented, value oriented and blend. The combination of orientation and market capitalization yields nine categories: Large-Cap Growth, Mid-Cap Growth and Small-Cap Growth; Large-Cap value, Mid-Cap Value and Small-Cap Value; Large-Cap Blend, Mid-Cap Blend and Small-Cap Blend. To make classification consistent with the multifactor models used in the study, Morningstar style box is used with modification: funds are evaluated in three size categories and in three book-to-market (BM) categories instead of in each of the nine categories the Morningstar style box produces.

Table B-1a. Morningstar Style Box

Market Cap
(size)




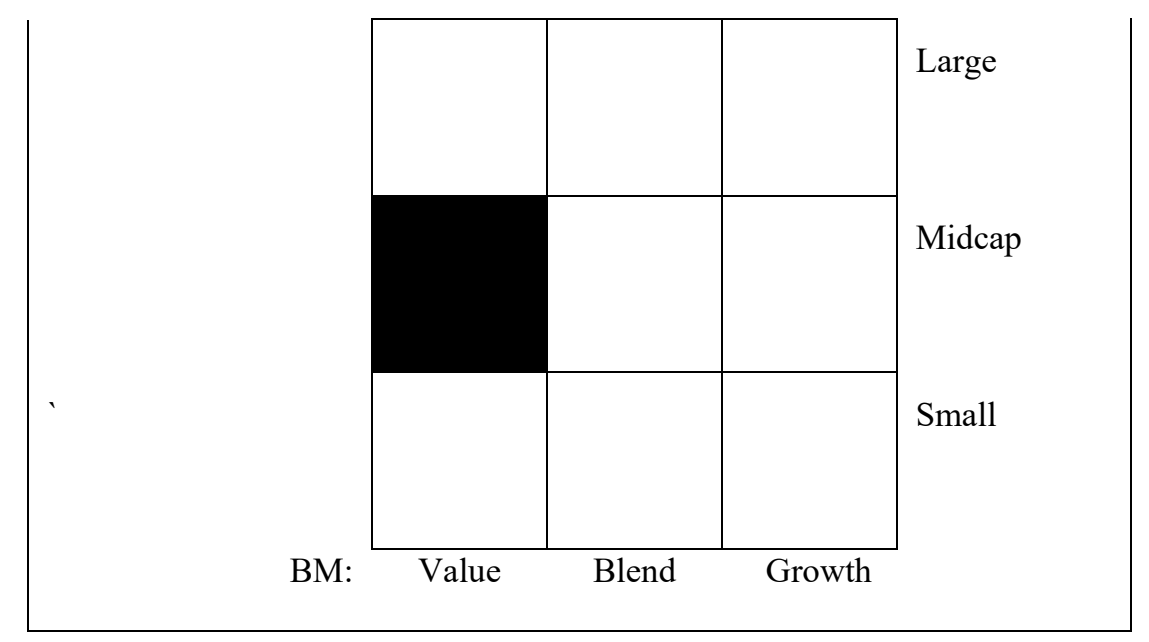

Table B-1b. Classification of funds for this study (based on Morningstar style box)

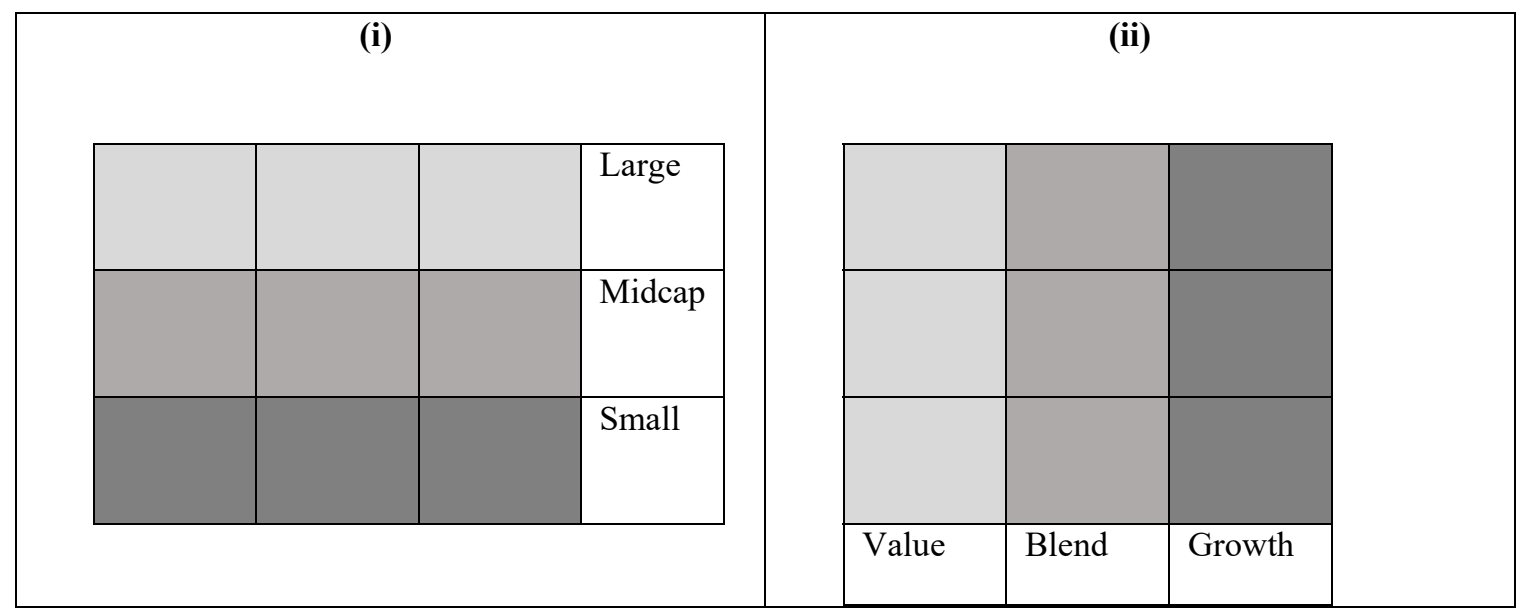

Table B-2. Appropriate benchmarks ${ }^{4}$ for each fund category.

\section{Fund Category}

By market capitalization (size)

Large Cap

\section{$\underline{\text { Relevant benchmark }}$}

Russell 1000 index 
Mid-Cap

Small Cap

By Book-to-Market ratio (BM)

Value

Blend

Growth
Russell Midcap index

Russell 2000 index

Russell 3000 Value index

Russell 3000 index

Russell 3000 Growth index

${ }^{1}$ Computed using Mutual Fund Historical Data. Retrieved from https://finance.yahoo.com/

${ }^{2}$ Monthly yield on Three-month Treasury Bill-Secondary Market rate. Retrieved from

https://fred.stlouisfed.org/series/TB3MS

${ }^{3}$ SMB, HML and MOM factors for the U.S. equity markets are obtained from Kenneth R. French Data Library.

Retrieved from http://mba.tuck.dartmouth.edu/pages/faculty/ken.french/data_library.html

${ }^{4}$ The FTSE Russell. Russell US Style Indexes. Retrieved from http://www.ftse.com/products/indices/russell-us-style 